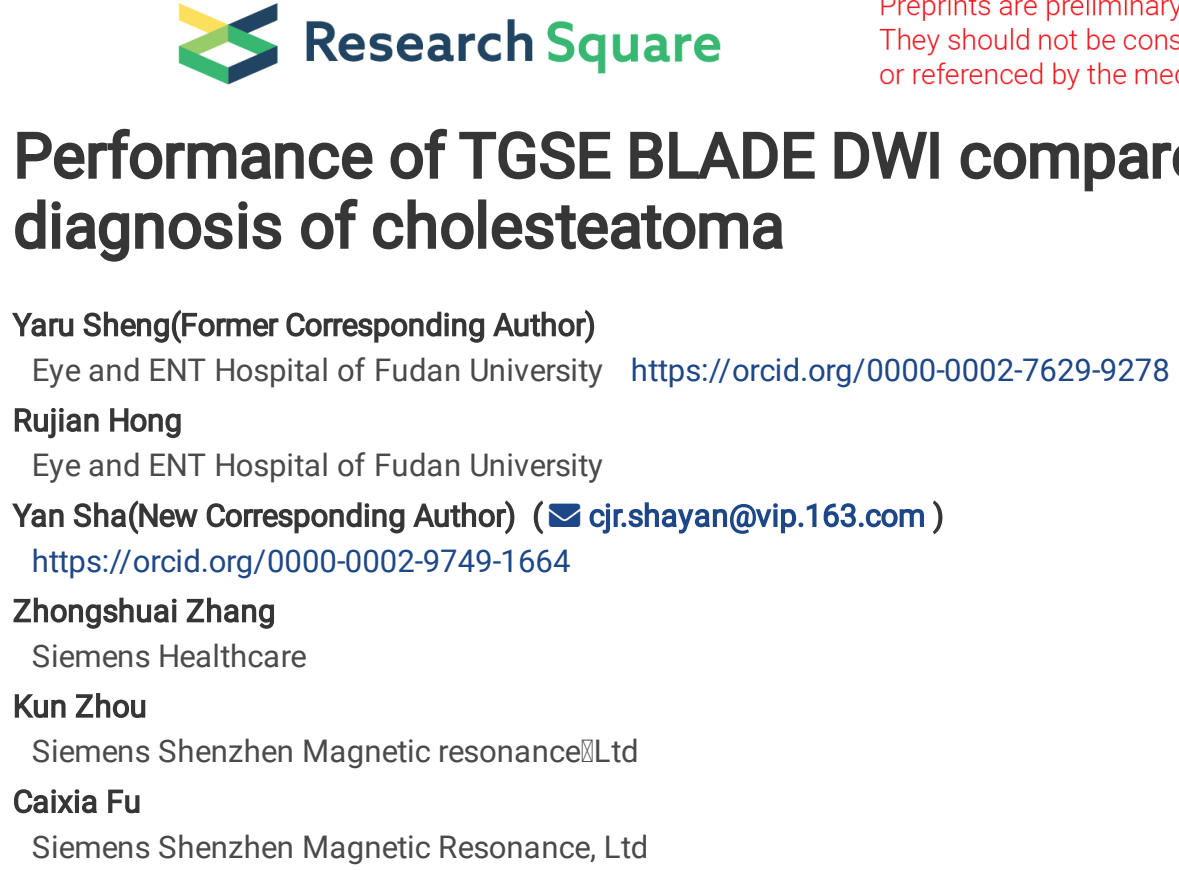

\title{
Performance of TGSE BLADE DWI compared with RESOLVE DWI in the diagnosis of cholesteatoma
}

Research article

Keywords: Cholesteatoma, Middle ear, Magnetic resonance imaging, TGSE BALDE DWI, RESOLVE DWI

Posted Date: March 2nd, 2020

DOI: https://doi.org/10.21203/rs.2.21206/v2

License: 두 (i) This work is licensed under a Creative Commons Attribution 4.0 International License. Read Full License

Version of Record: A version of this preprint was published at BMC Medical Imaging on April 19th, 2020. See the published version at https://doi.org/10.1186/s12880-020-00438-7. 


\section{Abstract}

Background :Based on its high resolution in soft tissue, MRI, especially diffusion-weighted imaging (DWI), is increasingly important in the evaluation of cholesteatoma. The purpose of this study was to evaluate the role of the 2D turbo gradient- and spin-echo (TGSE) diffusionweighted (DW) pulse sequence with the BLADE trajectory technique in the diagnosis of cholesteatoma at $3 T$ and to qualitatively and quantitatively compare image quality between the TGSE BLADE and RESOLVE methods.

Method:A total of 42 patients (23 males, 19 females; age range, 7-65 years; mean, 40.1 years) with surgically confirmed cholesteatoma in the middle ear were enrolled in this study. All patients underwent DWI (both a prototype TGSE BLADE DWI sequence and the RESOLVE DWI sequence) using a 3-T scanner with a 64-channel brain coil.

Qualitative imaging parameters (imaging sharpness, geometric distortion, ghosting artifacts, and overall imaging quality) and quantitative imaging parameters (apparent diffusion coefficient [ADC], signal-to-noise ratio [SNR], contrast, and contrast-to-noise ratio [CNR]) were assessed for the two diffusion acquisition techniques by two independent radiologists.

Result: A comparison of qualitative scores indicated that TGSE BLADE DWI produced less geometric distortion, fewer ghosting artifacts $(P<0.001)$ and higher image quality $(P<0.001)$ than were observed for RESOLVE DWI. A comparison of the evaluated quantitative image parameters between TGSE and RESOLVE showed that TGSE BLADE DWI produced a significantly lower SNR $(P<0.001)$ and higher parameter values (both contrast and CNR $(P<0.001)$ ) than were found for RESOLVE DWI.

The ADC $(P<0.001)$ was significantly lower for TGSE BLADE DWI $(0.763 \times 10-3 \mathrm{~s} / \mathrm{mm} 2)$ than RESOLVE DWI $(0.928 \times 10-3 \mathrm{~s} / \mathrm{mm} 2)$.

Conclusion: Compared with RESOLVE DWI, TGSE BLADE DWI significantly improved the image quality of cholesteatoma by reducing magnetic sensitive artifacts, distortion, and blurring. TGSE BLADE DWI is more valuable than RESOLVE DWI for the diagnosis of small-sized $(2 \mathrm{~mm})$ cholesteatoma lesions. However, TGSE BLADE DWI also has some disadvantages: the whole image intensity is slightly low, so that the anatomical details of the air-bone interface are not shown well, and this shortcoming should be improved in the future.

\section{Background}

Cholesteatoma is a benign, gradually expanding and destructive epithelial lesion of the temporal bone that results in the erosion of adjacent bony structures and can lead to various complications. ${ }^{1}$ In addition to its clinical features and otoscopic findings, the early diagnosis of cholesteatoma based on an imaging examinations, such as high-resolution computed tomography (CT) and magnetic resonance imaging (MRI), is important. From a surgical perspective, high-resolution CT remains the primary imaging technique for the diagnosis and characterization of cholesteatoma in the middle ear due to its high spatial resolution and good visualization of bone structures ${ }^{11,12}$. However, in terms of its disadvantages, it is difficult to distinguish cholesteatoma from granulation tissue, fibrous tissue, or fluid on high-resolution $\mathrm{CT}^{2}$. Based on the high resolution of soft tissue, MRI has gained increasing importance in the evaluation of cholesteatoma. Many studies have shown that MR diffusion-weighted imaging (DWI) has high sensitivity and specificity for identifying the presence of cholesteatoma due to its high keratin content ${ }^{13-15}$. However, conventional DWI uses an echo-planar imaging (EPI) trajectory to collect k-space data, and the obtained images (single-shot echo-planar DWI, SS EPI) may suffer from severe susceptibility artifacts at air-bone interfaces. Additionally, its image resolution is limited. Thus, it is difficult to use DWI in cases in which the lesion is closer than $5 \mathrm{~mm}^{3,4}$ from the distortion area. Compared with the EP DWI sequence, the non-echo-planar diffusion weighted imaging (non-EPI) DW imaging sequence produces thinner slices and has a higher imaging matrix, and it tends to produce fewer magnetic susceptibility artifacts but requires longer imaging times (multi-shot non-echo-planar DWI sequences require approximately 8 minutes), and non-EPI has higher sensitivity for detecting cholesteatoma and a lower misdiagnosis rate ${ }^{15,34-36}$.

Readout-segmented echo-planar imaging (RESOLVE) has been proposed to reduce image distortion. This method could significantly improve head and neck DWI by reducing echo spacing. Although RESOLVE DWI has a significantly improved image signal-to-noise ratio (SNR) and reduced image distortion, the partial volume effect and $\mathrm{T}^{*}$ blurring effect are not completely eliminated, and it is difficult to detect small lesions $(<2.5 \mathrm{~mm})^{6-8}$.

Periodically rotated overlapping parallel lines with enhanced reconstruction (PROPELLER) DWI is a nonecho planar fast spin-echo-based DWI sequence in which the central $k$-space is acquired in a rotating manner. The PROPELLER sequence is free of geometric distortion and susceptibility artifacts, but the scan time is long and imposes a high specific absorption rate (SAR), especially at high fields ${ }^{27-30}$.

The BLADE DWI technique has previously been reported to eliminate susceptibility artifacts by applying the 'blades' acquisition scheme in kspace $^{5}$. This non-EPI technique was further optimized by using a TGSE method to increase the SNR efficiency and achieve the detection of 
small $(<2.5 \mathrm{~mm})$ cholesteatomas while increasing the resolution to decrease susceptibility artifacts, thereby allowing differentiation from granulation tissue ${ }^{5}$. Houchun $\mathrm{H}$. et al. ${ }^{18}$ concluded that TGSE BLADE DWI exhibited less geometric distortion in the brain and reduced magnetic artifacts near the air-tissue interface than were achieved by conventional SE-EPI. However, the use of TGSE BLADE DWI in studying ear lesions has not yet been reported. L.M.J. Lips et al. ${ }^{19}$ found that applying non-EPI DWI for the detection of residual or recurrent cholesteatoma achieved better results at $3 \mathrm{~T}$ than at $1.5 \mathrm{~T}$. Hence, the purpose of this study was to evaluate the role of the TGSE BLADE DWI technique in the diagnosis of cholesteatoma at $3 \mathrm{~T}$ and qualitatively and quantitatively compare image quality between TGSE BLADE and RESOLVE protocols with the same scanning time (3 m46s).

\section{Methods}

\subsection{Patients}

In this study, a total of 42 patients (23 males, 19 females; age range, 7-65 years; mean, 40.1 years) with surgically confirmed cholesteatoma were enrolled, and patients with congenital cholesteatoma were excluded according to the clinical diagnosis from October 2018 to April 2019. Of the 42 patients, 37 had cholesteatoma in the middle and 5 had cholesteatoma in the external auditory canal. Clinicopathological results were the gold standard for all patients.

Ethics and Consent to Participate

The study was approved by the Review Committee of Eye \& ENT Hospital of Fudan University, and written informed consent was obtained from all patients.

\subsection{Imaging technique}

All patients underwent DWI (both a prototype TGSE BLADE DWI sequence and a commercially available RESOLVE DWI sequence) using a $3 \mathrm{~T}$ scanner (MAGNETOM Prisma, Siemens Healthcare, Erlangen, Germany) with a 64-channel brain coil. To better measure the transverse diameter of the lesion, especially the microlesion, axial TGSE and RESOLVE sequence images were selected.

The parameters for TGSE BLADE DWI were as follows: TR $/$ TE $=4000 / 62 \mathrm{~ms}$; slice thickness $/$ gap $=2 / 0.2 \mathrm{~mm}$; slices $=21$; bandwidth $=520$ $\mathrm{Hz} / \mathrm{Px}$; field of view $(\mathrm{FOV})=280 \times 280 \mathrm{~mm}^{2} ;$ matrix $=192 \times 192$; voxel size $=1.5 \times 1.5 \times 2.0 \mathrm{~mm}^{3}$; number of excitations $($ NEX $)=1$; diffusion mode $=4$ scan trace; $b=0,1000 \mathrm{~s} / \mathrm{mm}^{2} ;$ turbo factor $=13$; EPI factor $=3$; and data acquisition time $=3 \mathrm{~min} 46 \mathrm{~s}$. For RESOLVE DWI, the imaging parameters were as follows: TR $/ \mathrm{TE}=5020 / 53 \mathrm{~ms}$; slice thickness $/$ gap $=2 / 0.2 \mathrm{~mm}$; slices $=21$; bandwidth $=766 \mathrm{~Hz} / \mathrm{Px} ; \mathrm{FOV}=230$ $\times 230 \mathrm{~mm}$; matrix $=192 \times 192 ;$ voxel size $=1.2 \times 1.2 \times 2.0 \mathrm{~mm}$; diffusion mode $=4$ scan trace; $b=0,1000 \mathrm{~s} / \mathrm{mm}^{2} ;$ and data acquisition time = $3 \min 46 \mathrm{~s}$.

\subsection{Image assessment}

\subsubsection{Qualitative analysis of image quality}

All images obtained in the 42 included patients by TGSE or RESOLVE were evaluated by two radiologists with 10 years of experience in ear MRI evaluation.

Each observer randomly and blindly evaluated the images without knowing the type of DWI sequence and compared the two different DWI sequences using the side-by-side display method. A final decision was made based on mutual consultation when there was a divergence in the assessment results.

Qualitative evaluation of images obtained by TGSE and RESOLVE was performed according to four criteria: image sharpness, geometric distortion, ghosting artifacts, and overall image quality. Image sharpness was assessed on a scale from 1 to 3 . Both geometric distortion and ghosting artifacts were evaluated on a scale from 1 to 4 , and the evaluation of geometric distortion included two parts: the whole image and the lesion in the ear. Overall image quality was also graded on a scale of 1 to 5 . The detailed qualitative evaluation criteria are shown in Table 1. In Figure 1, images $C$ and $E$ show geometric deformations with a score of 4 (no distortion) and 2 (moderate distortion), respectively.

\subsubsection{Quantitative analysis of image quality}

The SNR, contrast and contrast-to-noise ratio (CNR) were the main evaluation criteria for the quantitative analysis of images obtained using the two sequence. The apparent diffusion coefficients (ADCs) of the two sequences were compared simultaneously. On the b1000 TGSE and RESOLVE images, the SNR of the lesions in the region of interest (ROI) was defined as the ratio of the mean signal intensity of the lesion $\left(\mathrm{S}_{\mathrm{ROI}}\right)$ to the standard deviation of the background noise $\left(\sigma_{\mathrm{BG}}\right)\left(\mathrm{SNR}=\mathrm{S}_{\mathrm{RO} /} / \sigma_{\mathrm{BG}}\right)^{9}$. The $\mathrm{SNR}$ of the brainstem was calculated by the same 
method as follows: $\mathrm{SNR}=\mathrm{S}_{\mathrm{B}} / \sigma_{\mathrm{BG}}$. Contrast was defined as the ratio of the signal intensity of the lesion $\left(\mathrm{S}_{\mathrm{ROI}}\right)$ to the signal intensity of the brainstem $\left(\mathrm{S}_{\mathrm{B}}\right)$ on the b1000 map (contrast $\left.=\mathrm{S}_{\mathrm{ROI}} / \mathrm{S}_{\mathrm{B}}\right)$.

The CNR was defined as the difference between the $S_{\mathrm{ROI}}$ and $\mathrm{S}_{\mathrm{B}}$ divided by the standard deviation in the lesion ROI ( $\left.\sigma_{\mathrm{ROI}}\right)$ and the brainstem $\mathrm{ROI}\left(\sigma_{\mathrm{B}}\right)^{6-8,10}$, as follows:

The ROI of the lesion on the b1000 and ADC maps was manually drawn as $1 \mathrm{~mm}^{2}$ at the level of the maximum diameter of the lesion, and the corresponding signal intensity and standard deviation were automatically generated on the MRI workstation. The ROI of the brainstem was defined by selecting $10 \mathrm{~mm}^{2}$ of the brainstem, and the signal intensity and standard deviation of each ROI were automatically generated. A circular ROI of $10 \mathrm{~mm}^{2}$ was set in the background on the b1000 map for all patients, and the standard deviation of the ROI was automatically generated. In selecting the ROI, areas affected by susceptible artifacts or volume effects were avoided.

The parameters were measured independently and randomly by the two raters at an interval of 2 weeks. The mean value of the two measurements was selected as the final data for further analysis. For brain tissue evaluated on DWI sequences, the diagnostic criterion for cholesteatoma was a very high signal intensity that corresponded to limited diffusion on the ADC maps ${ }^{(3,20)}$. The sizes of all lesions were determined on T2-weighted imaging (T2WI) based on the size and location of the lesions observed on the TGSE and RESOLVE sequence images and the premise of avoiding artifacts at air-bone interfaces as much as possible.

\subsection{Statistical analysis}

All statistical analyzes and plots were performed and created using the SPSS 24.0 software package (Chicago, IL, USA), and P < 0.05 was considered statistically significant. The normality of all measurements obtained using the TGSE and RESOLVE sequences was tested using the Shapiro-Wilk test. Significant differences in qualitative parameters between TGSE and RESOLVE DWI were determined using the Wilcoxon rank-sum test, and significant differences in quantitative parameters were determined using the paired t-test. The interreader correlation of the $A D C$ as a quantitative index was evaluated using the intraclass correlation coefficient (ICC). The range of the ICC coefficient was set from 0 to 1.00, and the ICC was defined as follows: < 0.40, poor; 0.41-0.60, moderate; 0.61- 0.80, good; >0.81, excellent ${ }^{16,17}$. The mean ADCs of the lesions and brainstem measured by the two observers were further calculated, and the differences between them were assessed by paired ttest.

\section{Results}

The lesions were clearly displayed on the TGSE and RESOLVE sequence images obtained in 40 patients among the 42 cases. In only two cases, the RESOLVE sequence images produced more magnetic susceptibility artifacts because the lesion was too small (1.9 mm), and it was difficult to distinguish the lesions from the artifacts, while the TGSE sequence images showed the lesions clearly (Figure 4).

\subsection{Qualitative analysis of image quality}

Comparison of the qualitative scores indicated that TGSE BLADE DWI produced less geometric distortion and ghosting artifacts ( $<0.001)$ and had higher image quality $(P<0.001)$ than were found for RESOLVE DWI. The average TGSE and RESOLVE scores were as follows: geometric distortion (whole), 3.97 \pm 0.15 and 3.26 \pm 0.26 , respectively ( $P<0.001$ ); geometric distortion (lesion), $3.95 \pm 0.21$ and $3.64 \pm 0.48$, respectively $(P<0.001)$; ghosting artifacts, $3.92 \pm 0.26$ and $3.07 \pm 0.55$, respectively $(P<0.001)$; and overall image quality, $4.85 \pm 0.35$ and 4.16 \pm .69 , respectively. Both TGSE BLADE DWI and RESOLVE DWI had nearly perfect image sharpness $(P=0.23 ; 2.85 \pm 0.35$ versus $2.73 \pm 0.49)$. Table 1 shows a comparison of the qualitative parameter scores for TGSE BLADE DWI and RESOLVE DWI, and Figure 2 shows the distributions of the qualitative scores obtained using TGSE BLADE DWI and RESOLVE DWI. As shown in Figure 1, axial TGSE DWI precisely defined the cholesteatoma lesion in the right middle ear without geometric distortion or ghosting artifacts, whereas RESOLVE DWI showed significant artifacts at the air-bone interface (between the mastoid, i.e., the location of the cholesteatoma lesion, and the nasal sinus).

\subsection{Quantitative analysis of image quality}

Comparison of the evaluated quantitative image parameters between TGSE and RESOLVE showed significant differences between the two groups. TGSE BLADE DWI produced a significantly lower SNR $(P<0.001)$ and higher parameter values (both contrast and CNR $(P<0.001)$ ) than were found for RESOLVE DWI. The results of the statistical analysis are as follows (Table 1): SNR of cholesteatoma, $102.3 \pm 32.2$ versus 493.7 \pm 241.6 , respectively $(P<0.001)$; SNR of brainstem, $59.1 \pm 15.5$ versus $289.8 \pm 140.9$, respectively $(P<0.001)$; contrast, $1.79 \pm 0.35$ versus $1.62 \pm 0.44$, respectively $(P=0.005)$; and $C N R, 1.62 \pm 0.44$ versus $2.7 \pm 2.6$, respectively $(P<0.001)$. 
In terms of the measurement and evaluation of the ADC, values were measured in 40 cases, as the lesions were too small to be measured on the ADC maps in 2 cases. Excellent interreader agreement was obtained. Detailed interreader ICCs are shown in Table 2. All ADCs were measured twice by the two observers, and the average values were taken as the basis for further statistical analysis. As shown in Table 2 , there was a significant difference in the ADC of cholesteatoma between TGSE BLADE and RESOLVE DWI (P<0.01). The mean ADC of the cholesteatoma measured on TGSE $\left(0.763 \times 10^{-3} \mathrm{~s} / \mathrm{mm}^{2}\right)$ BLADE DWI was significantly lower than that measured on RESOLVE $\left(0.928 \times 10^{-3}\right.$ $\left.\mathrm{s} / \mathrm{mm}^{2}\right)$ DWI $(\mathrm{P}<0.01)$. Similarly, the ADC of the brainstem measured on TGSE $\left(0.498 \times 10^{-3} \mathrm{~s} / \mathrm{mm}^{2}\right)$ BLADE DWI was lower than that measured on RESOLVE $\left(0.773 \times 10^{-3} \mathrm{~s} / \mathrm{mm}^{2}\right)$ DWI $(\mathrm{P}<0.01)$. The box plot in Figure 3 shows the distributions of the lesion and brainstem ADCs measured on TGSE BLADE and RESOLVE DWI. There was no significant difference in ADC values between the 5 patients with cholesteatoma of the external auditory canal and the 35 patients with cholesteatoma of the middle ear between the TGSE ( $P=0.236)$ and RESOLVE $(P=0.127)$ images.

In this study, the measurement results in terms of lesion size were evaluated in 42 patients and showed that TGSE BLADE DWI showed small lesions more clearly than was achieved by RESOLVE DWI. Compared with RESOLVE, TGSE had much better image quality at the air-bone interface (nasal sinus, middle ear, mastoid) and significantly fewer ghosting artifacts and distortion. Furthermore, as shown in Figure 4, axial TGSE BLADE DWI could completely and clearly show a small lesion (1.9 mm in width) located in the left tympanic cavity with less geometric distortion than was observed using RESOLVE DWI.

\section{Discussion}

DWI is increasingly applied for the evaluation of various diseases in many areas of the body. Conventional DWI (SS-EPI) is often used in head and neck diseases; however, due to interference by the T2* blurring effect and the susceptibility artifacts of various tissues produced by nonmovement, the image quality of conventional DWI (SS-EPI) is usually not satisfactory ${ }^{4 \llbracket 26}$. Many studies ${ }^{(3,21,22)}$ have concluded that the size limit of cholesteatoma on EPI DWI is $5 \mathrm{~mm}$ and that smaller cholesteatoma lesions are easily missed on DW EPI. Compared with conventional SS-EPI DWI, RESOLVE has significantly better image quality due to its low susceptibility-based image distortion and T2* blurring and its robust correction for motion-induced phase artifacts ${ }^{23}$. RESOLVE DWI is therefore more widely used in head and neck diseases than SS-EPI DWI is. However, RESOLVE still has some shortcomings that need to be resolved; these include image artifacts and distortion (air-bone interface) that cannot be completely eliminated and a low diagnostic rate of small lesions $(<2.5 \mathrm{~mm})^{6-8,14,24}$.

To the best of our knowledge, TGSE is a new technique, and the use of DWI in head and neck diseases has not previously been reported in the literature. The basic imaging principles of the TGSE BLADE technique were introduced by Li et al ${ }^{25}$. In this study, compared to RESOLVE DWI, TGSE BLADE DWI significantly improved the image quality in cases of cholesteatoma by reducing susceptibility artifacts, distortion and blurring when applied at the same scanning time (3 min $46 \mathrm{~s}$ ). Moreover, TGSE BLADE DWI may be more valuable than RESOLVE DWI for the diagnosis of small cholesteatoma lesions $(<2 \mathrm{~mm})$.

Qualitative analysis of image quality showed that almost no geometric distortion or ghosting artifacts were observed in the TGSE images, while the RESOLVE images contained obvious geometric distortion, mostly in the nasal cavity and mastoid, in 8 of 42 (20\%) cases. Moreover, serious artifacts were observed in the RESOLVE images in 5 (12\%) cases. Hu ${ }^{18}$ et al. also demonstrated that TGSE BLADE DWI produced less geometric distortion in the brain and signal pile-up in highly susceptible areas than was found for conventional SE-EPI. The image quality of TGSE BLADE has also been significantly improved. In the quantitative analysis of image quality, TGSE BLADE DWI produced higher contrast and a higher CNR than was observed for RESOLVE DWI. These were prospective results due to the lack of previous reports on TGSE BLADE DWI.

This study shows that there is a significant difference in the ADC between the TGSE BLADE and RESOLVE sequences, with a significantly lower ADC of the cholesteatoma $(P<0.01)$ and brainstem $(P<0.01)$ found when using TGSE BLADE DWI than RESOLVE DWI. The average ADC for cholesteatoma on RESOLVE DWI was $0.928 \times 10^{-3} \mathrm{~s} / \mathrm{mm}^{2}$, which is consistent with the cholesteatoma ADC $\left(0.7-1.0 \times 10^{-3} \mathrm{~s} / \mathrm{mm}^{2}\right)$ previously reported in the literature ${ }^{31-33}$. The mean ADC of the cholesteatoma and brainstem on TGSE BLADE DWI was $0.763 \times 10^{-3} \mathrm{~s} / \mathrm{mm}^{2}$ and $0.498 \times 10^{-3} \mathrm{~s} / \mathrm{mm}^{2}$, respectively. These findings demonstrate that the ADC obtained in our study should provide an auxiliary basis for more clinical applications of TGSE BLADE DWI in the future.

However, there are also some limitations to our study: the number of patients included in this study was relatively small, and the error caused by manual measurement could not be eliminated. This may have affected the accuracy of the true range of the ADC on TGSE BLADE DWI. Moreover, TGSE BLADE DWI is not without its disadvantages. The overall image SNR of TGSE is slightly lower than that achieved by RESOLVE, mainly because placement of the gradient echo with T2* decay effects in the center of k-space reduces the image quality of TGSE, consistent with a previous pediatric brain study reported by $\mathrm{Ui}^{26}$ et al. 


\section{Conclusion}

In conclusion, TGSE BLADE DWI produced better image quality than was achieved by RESOLVE DWI in the diagnosis of cholesteatoma. TGSE BLADE DWI was also superior to RESOLVE DWI in terms of image distortion, artifacts and lesion conspicuity. In addition, TGSE BLADE DWI appears to be more effective than RESOLVE DWI in detecting small lesions.

\section{Declarations}

Ethics approval and consent to participate

This study was approved by the Review Committee of Eye \& ENT Hospital of Fudan University, and written informed consent was obtained from all patients. For participants under 16 years old, written informed consent was obtained from a parent or guardian.

\section{Consent for publication}

All authors of this article have agreed to publish this article.

Availability of data and material

The datasets used and analyzed in the current study are available from the corresponding author on reasonable request.

\section{Competing interests}

The authors declare that they have no competing interests.

\section{Funding}

The authors state that this work has not received any funding.

\section{Authors' contributions}

ZZS and SYR designed this study

SYR collected patient data and was a major contributor to writing the manuscript.

HRJ and SY analyzed and evaluated two kinds of sequential images.

ZK and FCX provide guidance on magnetic resonance techniques.

All authors read and approved the final manuscript.

Acknowledgements

Not applicable

\section{Abbreviations}

ADC: apparent diffusion coefficient

SNR: signal-to-noise ratio

CNR: contrast-to-noise ratio

SAR: specific absorption rate

ROI: region of interest

ICC: intraclass correlation coefficient 


\section{References}

1. Louw L. Acquired cholesteatoma pathogenesis: stepwise explanations. The Journal of laryngology and otology. Jun 2010;124(6):587593.

2. Yates PD, Flood LM, Banerjee A, Clifford K. CT scanning of middle ear cholesteatoma: what does the surgeon want to know? The British journal of radiology. Oct 2002;75(898):847-852.

3. Vercruysse JP, De Foer B, Pouillon M, Somers T, Casselman J, Offeciers E. The value of diffusion-weighted MR imaging in the diagnosis of primary acquired and residual cholesteatoma: a surgical verified study of 100 patients. European radiology. Jul 2006;16(7):14611467.

4. De Foer B, Vercruysse JP, Pilet B, et al. Single-shot, turbo spin-echo, diffusion-weighted imaging versus spin-echo-planar, diffusionweighted imaging in the detection of acquired middle ear cholesteatoma. AJNR. American journal of neuroradiology. Aug 2006;27(7):1480-1482.

5. Dhepnorrarat RC, Wood B, Rajan GP. Postoperative non-echo-planar diffusion-weighted magnetic resonance imaging changes after cholesteatoma surgery: implications for cholesteatoma screening. Otology \& neurotology : official publication of the American Otological Society, American Neurotology Society [and] European Academy of Otology and Neurotology. Jan 2009;30(1):54-58.

6. Bogner W, Pinker-Domenig K, Bickel H, et al. Readout-segmented echo-planar imaging improves the diagnostic performance of diffusionweighted MR breast examinations at 3.0 T. Radiology. 2012;263(1):64-76.

7. Xu, X; Wang, Y; Hu, H; Su, G; Liu, H;(2017). Readout-segmented echo-planar diffusion-weighted imaging in the assessment of orbital tumors: comparison with conventional single-shot echo-planar imaging in image quality and diagnostic performance. Acta Radiologica, 58(12), 1457-1467.

8. Menglong Zhao; Zhuang Liu; Yan Shaet al. Readout-segmented echo-planar imaging in the evaluation of sinonasal lesions: A comprehensive comparison of image quality in single-shot echo-planar imaging. [J]. Magn Reson Imaging, 2016, 34: 166-72.

9. Kaufman L,Kramer D M,Crooks L E et al. Measuring signal-to-noise ratios in MR imaging.[J] .Radiology, 1989, $173: 265-7$.

10. Xiaomei Song, Pogue Brian W,Jiang Shudong et al. Automated region detection based on the contrast-to-noise ratio in near-infrared tomography.[J] .Appl Opt, 2004, 43: 1053-62.

11. Yamashita Koji,Yoshiura Takashi,Hiwatashi Akio et al. Contributing factors in the pathogenesis of acquired cholesteatoma: size analysis based on MDCT.[J] .AJR Am J Roentgenol, 2011, 196: 1172-5.

12. Razek Ahmed Abdel Khalek Abdel,Ghonim Mohamed Rashad,Ashraf Bassem,Computed Tomography Staging of Middle Ear Cholesteatoma.[J] .Pol J Radiol, 2015, 80: 328-33.

13. De Foer Bert,Vercruysse Jean-Philippe,Bernaerts Anja et al. Middle ear cholesteatoma: non-echo-planar diffusion-weighted MR imaging versus delayed gadolinium-enhanced T1-weighted MR imaging-value in detection.[J] .Radiology, 2010, 255: 866-72.

14. Algin O,Aydın H,Ozmen E et al. Detection of cholesteatoma: High-resolution DWI using RS-EPI and parallel imaging at 3 tesla.[J] .J Neuroradiol, 2017, 44: 388-394.

15. Henninger Benjamin,Kremser Christian,Diffusion weighted imaging for the detection and evaluation of cholesteatoma.[J] .World J Radiol, 2017, 9: 217-222.

16. Tyng Chiang J,Chojniak Rubens,Pinto Paula N V et al. Conformal radiotherapy for lung cancer: interobservers' variability in the definition of gross tumor volume between radiologists and radiotherapists.[J] .Radiat Oncol, 2009, 4: 28.

17. Fleiss JL, Cohen J. The equivalence of weighted kappa and the intraclass correlation coefficient as measures of reliability. Educ Psychol Meas 1973;33:613-619.

18. Houchun H,McAllister Aaron S,Jin Ning et al. Comparison of 2D BLADE Turbo Gradient- and Spin-Echo and 2D Spin-Echo Echo-Planar Diffusion-Weighted Brain MRI at 3 T: Preliminary Experience in Children.[J] .Acad Radiol, 2019, undefined: undefined.

19. Lips L M J,Nelemans P J,Theunissen F M D et al. The diagnostic accuracy of 1.5 T versus 3 T non-echo-planar diffusion-weighted imaging in the detection of residual or recurrent cholesteatoma in the middle ear and mastoid.[J] .J Neuroradiol, 2019, undefined: undefined.

20. Nevoux J,Lenoir M,Roger G et al. Childhood cholesteatoma.[J] .Eur Ann Otorhinolaryngol Head Neck Dis, $2010,127: 143-50$.

21. De Foer Bert,Vercruysse Jean-Philippe,Bernaerts Anja et al. The value of single-shot turbo spin-echo diffusion-weighted MR imaging in the detection of middle ear cholesteatoma.[J] .Neuroradiology, 2007, 49: 841-8.

22. Venail F,Bonafe A,Poirrier V et al. Comparison of echo-planar diffusion-weighted imaging and delayed postcontrast T1-weighted MR imaging for the detection of residual cholesteatoma.[J] .AJNR Am J Neuroradiol, 2008, 29: 1363-8. 
23. Porter David A,Heidemann Robin M,High resolution diffusion-weighted imaging using readout-segmented echo-planar imaging, parallel imaging and a two-dimensional navigator-based reacquisition.[J] .Magn Reson Med, 2009, 62: 468-75.

24. Williams Marc T,Ayache Denis,Alberti Corinne et al. Detection of postoperative residual cholesteatoma with delayed contrast-enhanced MR imaging: initial findings.[J] .Eur Radiol, 2003, 13: 169-74.

25. Zhiqiang Li,Pipe James G,Lee Chu-Yu et al. X-PROP: a fast and robust diffusion-weighted propeller technique.[J] .Magn Reson Med, 2011, 66: 341-7.

26. Attenberger UI, Runge VM, Stemmer A, Williams KD, et al. Diffusion weighted imaging: a comprehensive evaluation of a fast spin echo DWI sequence with BLADE (PROPELLER) k-space sampling at $3 \mathrm{~T}$, using a 32-channel head coil in acute brain ischemia.Invest Radiol. 2009 Oct;44(10):656-61.

27. Kida Ikuhiro,Ueguchi Takashi,Matsuoka Yuichiro et al. Comparison of Diffusion-Weighted Imaging in the Human Brain Using ReadoutSegmented EPI and PROPELLER Turbo Spin Echo With Single-Shot EPI at 7 T MRI.[J] .Invest Radiol, 2016, 51: 435-9.

28. Forbes K P,Pipe J G,Bird C R et al. PROPELLER MRI: clinical testing of a novel technique for quantification and compensation of head motion.[J] .J Magn Reson Imaging, 2001, 14: 215-22.

29. Pipe James G,Farthing Victoria G,Forbes Kirsten P,Multishot diffusion-weighted FSE using PROPELLER MRI.[J] .Magn Reson Med, 2002, 47: 42-52.

30. Kim T, Baek M,Park J et al. Comparison of DWI Methods in the Pediatric Brain: PROPELLER Turbo Spin-Echo Imaging Versus ReadoutSegmented Echo-Planar Imaging Versus Single-Shot Echo-Planar Imaging.[J] .AJR Am J Roentgenol, 2018, $210: 1352-1358$.

31. Russo Camilla,Elefante Andrea,Di Lullo Antonella M et al. ADC Benchmark Range for Correct Diagnosis of Primary and Recurrent Middle Ear Cholesteatoma.[J] .Biomed Res Int, 2018, 2018: 7945482.

32. Algin O,Aydın H, Ozmen E et al. Detection of cholesteatoma: High-resolution DWI using RS-EPI and parallel imaging at 3 tesla.[J] .J Neuroradiol, 2017, 44: 388-394.

33. Thiriat S, Riehm S, Kremer S et al. Apparent diffusion coefficient values of middle ear cholesteatoma differ from abscess and cholesteatoma admixed infection.[J] .AJNR Am J Neuroradiol, 2009, 30: 1123-6.

34. Lingam Ravi K,Nash Robert, Majithia Anooj et al. Non-echoplanar diffusion weighted imaging in the detection of post-operative middle ear cholesteatoma: navigating beyond the pitfalls to find the pearl.[J] .Insights Imaging, 2016, 7: 669-78.

35. Elefante A, Cavaliere M, Russo C et al. Diffusion weighted MR imaging of primary and recurrent middle ear cholesteatoma: an assessment by readers with different expertise.[J] .Biomed Res Int, 2015, 2015: 597896.

36. Schwartz K M, Lane J I,Bolster B D et al. The utility of diffusion-weighted imaging for cholesteatoma evaluation.[J] .AJNR Am J Neuroradiol, 2011, 32: 430-6.

\section{Tables}


Table 1. Qualitative criteria for comparing image quality of TGSE and RESOLVE (b1000) sequences. Image sharpness (anatomical structures: nasal sinus, ear, brain)

1. Completely unclear

2. Generally visible

3. Clearly visible

Geometric distortion
1. Severe distortion
2. Moderate distortion
3. Mild distortion
4. No distortion

Ghosting artifacts (interface of cholesteatoma and the temporal lobe)

1. Severe artifact, unable to distinguish lesions and normal tissues

2. Moderate artifact, part of the lesion can be distinguished from normal tissues

3. Mild artifact, no impact on lesion diagnosis

4. No artifact
Overall image quality
1. Nondiagnostic
2. Barely diagnosis
3. Diagnostic
4. Good
5. Excellent

Table 3. Comparison of ADC values between two observers

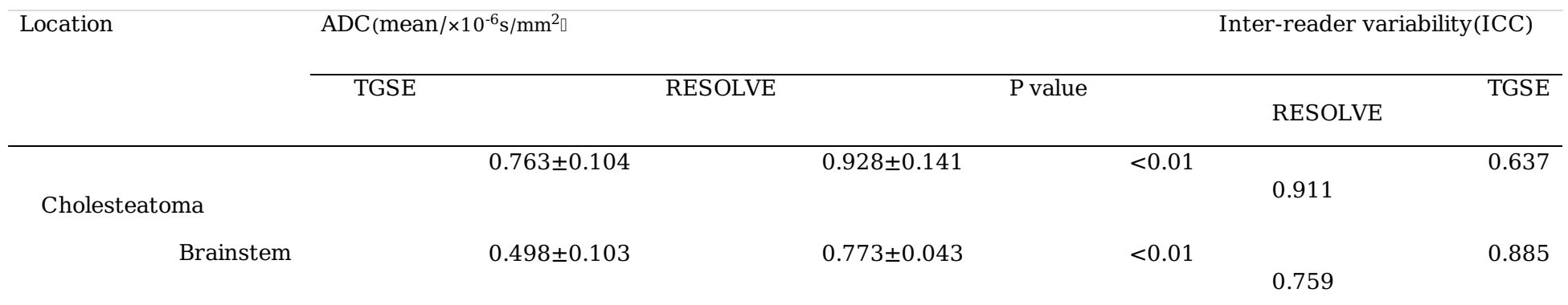

\section{Figures}


Table 2. Comparison of results of qualitative parameter evaluation between TGSE and RESOLVE images (42 patients).

$\begin{array}{llll}\text { Parameters TGSE RESOLVE Palue } & \text { T }\end{array}$

Qualitative parameters

$\begin{array}{lccc}\text { 1.Imaging sharpness } & 2.85 \pm 0.35 & 2.73 \pm 0.49 & 0.23 \\ & 3.97 \pm 0.15 \text { (whole) } & 3.26 \pm 0.76 \text { (whole) } & <0.01 \\ \text { 2.Geometric distortion } & 3.95 \pm 0.21 \text { (lesion) } & 3.64 \pm 0.48 \text { (lesion) } & <0.01 \\ \text { 3.Ghosting artifacts } & 3.92 \pm 0.26 & 3.02 \pm 0.55 & <0.01 \\ \text { 4.Overall imaging quality } & 4.85 \pm 0.35 & 4.16 \pm 0.69 & <0.01\end{array}$

Quantitative parameters

$1 . \operatorname{SNR}\left(\mathrm{b}=1000 \mathrm{~s} / \mathrm{mm}^{2}\right)$

\begin{tabular}{|c|c|c|c|}
\hline Lesion & $102.3 \pm 32.2$ & $493.7 \pm 241.6$ & $<0.01$ \\
\hline Brainstem & $59.1 \pm 15.5$ & $289.8 \pm 140.9$ & $<0.01$ \\
\hline 2.Contrast & $1.79 \pm 0.35$ & $1.62 \pm 0.44$ & $=0.005$ \\
\hline 3. $\mathrm{CNR}\left(\mathrm{b}=1000 \mathrm{~s} / \mathrm{mm}^{2}\right)$ & $4.33 \pm 2.8$ & $2.7 \pm 2.6$ & $<0.01$ \\
\hline
\end{tabular}

ratio

Note: SNR, signal to noise ratio; CNR, contrast to noise

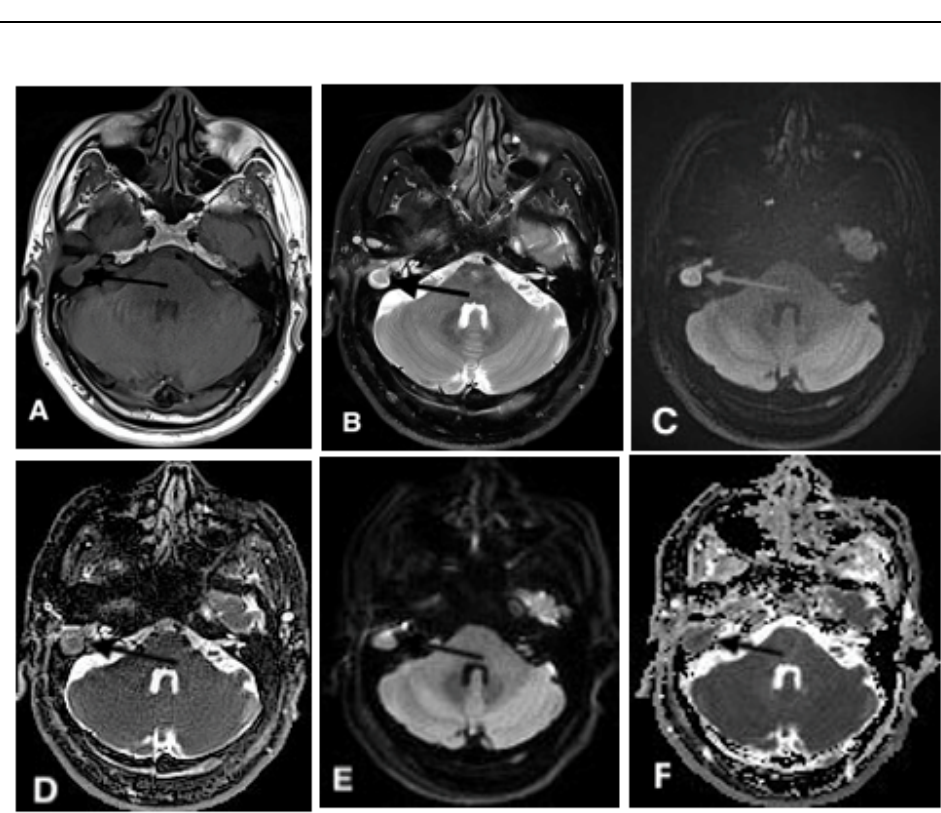

Figure 1

A 51-year-old male with primary cholesteatoma in the right middle ear confirmed by right mastoidectomy. Axial T1WI (A) and T2WI (B) showed the anatomical location of the cholesteatoma (arrow) in the right middle ear. (C, E): TGSE BLADE (b1000) and RESOLVE (b1000) DWI showed a restricted diffusion lesion (high signal) in the right mastoid bone. (D, F): The ADC on TGSE BLADE and RESOLVE DWI was $0.689 \times 10-3 \mathrm{~s} / \mathrm{mm} 2$ and $0.791 \times 10-3 \mathrm{~s} / \mathrm{mm} 2$, respectively. However, the cholesteatoma lesion (arrow) was not as clear on RESOLVE DWI as on TGSE DWI. Moreover, the structures of the nasal cavity were obviously distorted on the RESOLVE b1000 (E) and ADC (F) maps, whereas 
almost no distortion was observed on the TGSE b1000 and ADC maps. Images C and E show the geometric deformation values of the entire image for images with a score of 4 (no distortion) and 2 (moderate distortion).

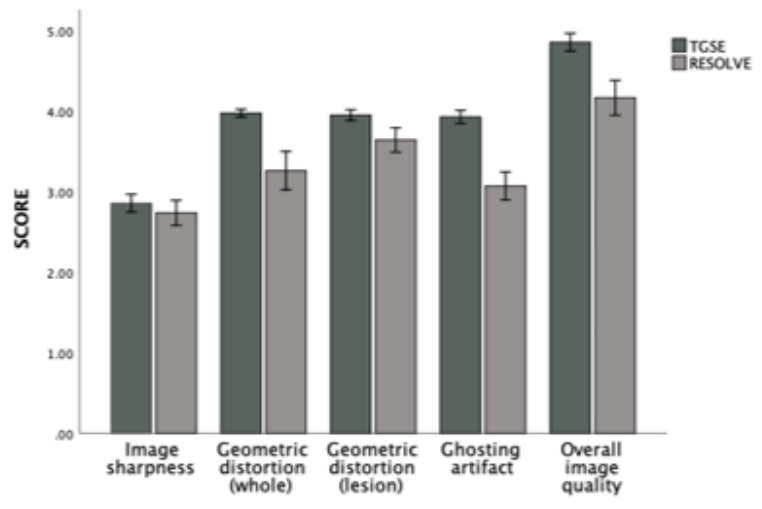

\section{Figure 2}

Bar chart showing a comparison of the qualitative imaging parameters between TGSE BLADE and RESOLVE DWI.

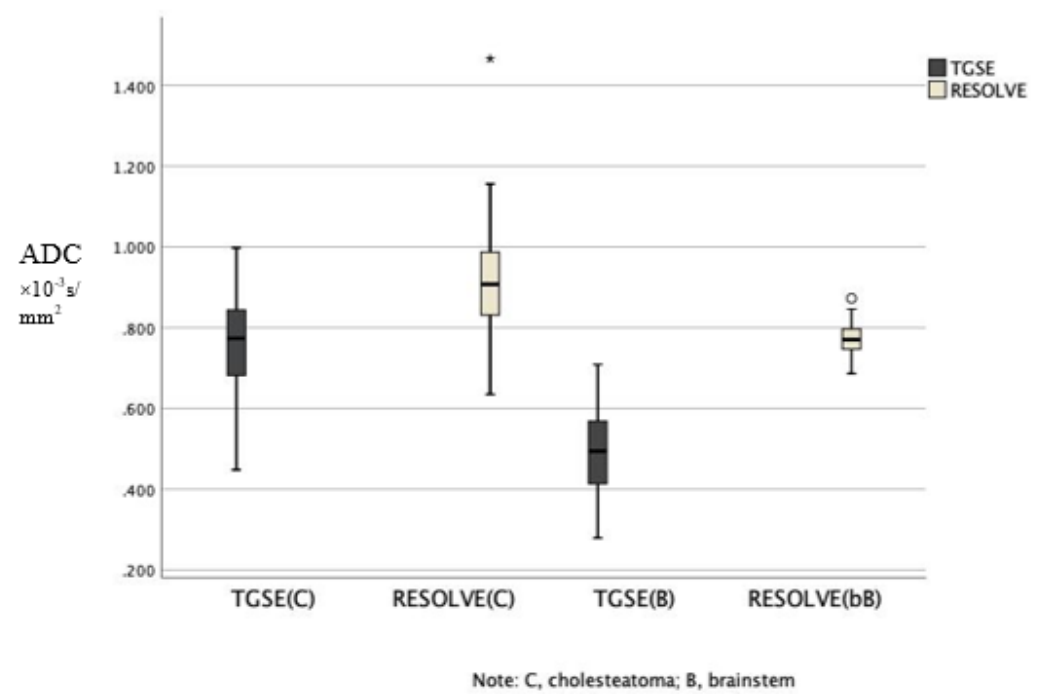

\section{Figure 3}

Box plot of the ADC of the cholesteatoma and brainstem showing significant differences in the ADC between TGSE and RESOLVE. The ADC values of the cholesteatoma measured on TGSE and RESOLVE DWI were $0.763 \times 10-6 \mathrm{~s} / \mathrm{mm} 2$ and $0.928 \times 10-3 \mathrm{~s} / \mathrm{mm} 2$, respectively. The ADC values of the brainstem measured on TGSE and RESOLVE DWI were $0.498 \times 10-3 \mathrm{~s} / \mathrm{mm} 2$ and $0.773 \times 10-3 \mathrm{~s} / \mathrm{mm} 2$, respectively. 


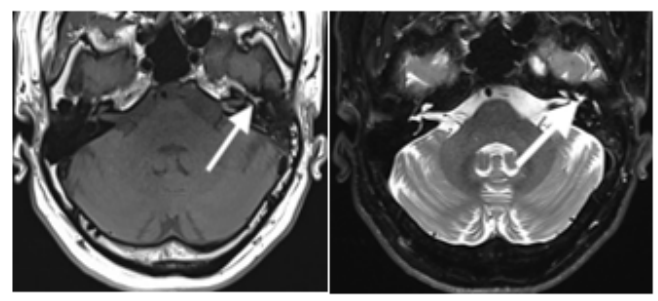

a

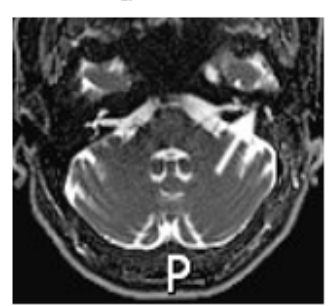

d b

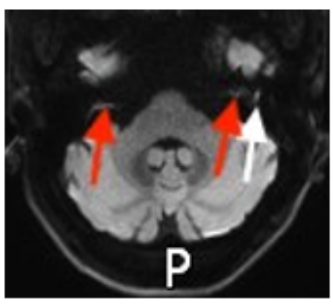

e

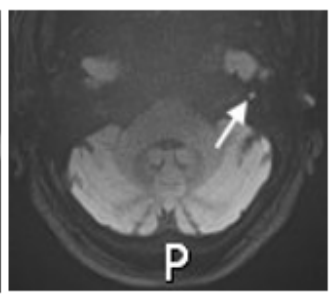

C

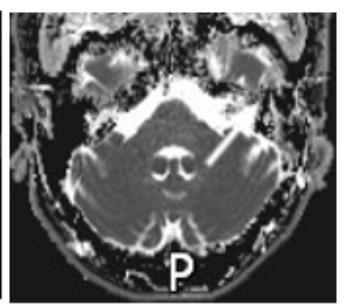

f

\section{Figure 4}

A 44-year-old male with a small cholesteatoma (1.9 mm width) in the left tympanic cavity (white arrow). (a): Axial T1WI. (b): Axial T2WI showing the structure of the small cholesteatoma (white arrow). (c): Axial TGSE BLADE DWI (b1000) clearly showing a markedly high signal intensity for the small cholesteatoma (white arrows) without artifacts. (e): Axial RESOLVE DWI (b1000) showing the high signal intensity of a small lesion (white arrow) with light geometric distortion and the bilateral middle ear mastoid process with a few artifacts (red arrow). (d, $\mathrm{f}$ ): The ADC values on TGSE BLADE and RESOLVE DWI were $0.737 \times 10-3 \mathrm{~s} / \mathrm{mm} 2$ and $0.984 \times 10-3 \mathrm{~s} / \mathrm{mm} 2$, respectively.

\section{Supplementary Files}

This is a list of supplementary files associated with this preprint. Click to download.

- formula1.PNG 